\section{A 2 Year Old Female Child with Huge Vaginal Rhabdomyosarcoma}

Received: January 15, 2016; Accepted: January 18, 2016; Published: January 21, 2016

Figure 1 shows polypoidal growth protruding through the vagina with no evidence of invasion of surrounding structures in a 2 year old female child. Provisional diagnosis was made as vaginal rhabdomyosarcoma which later on confirmed as botryoid rhabdomyosarcoma through biopsy. Contrast Enhanced Computed Tomography (CECT) showed multiple growths (Figure 2).

We achieved a satisfactory outcome in short-term period with excision followed by VAC regimen chemotherapy (i.e. three drug combination which includes vincristine and dactinomycin (also known asactinomycin-D) and cyclophosphamide).

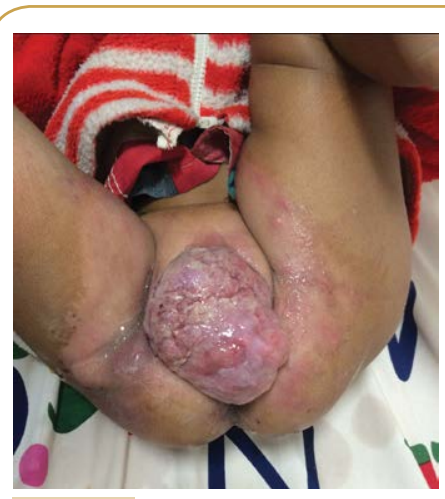

Figure 1 Image showing botryoid rhabdomyosarcoma in a 2 year old female child.

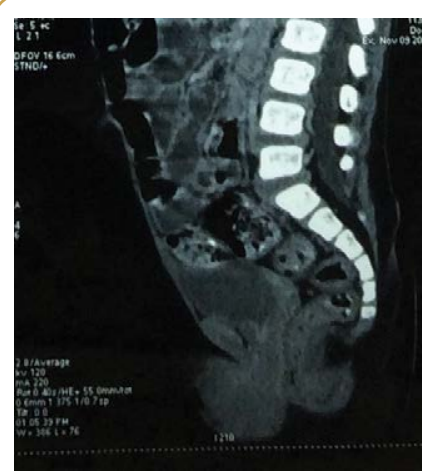

Figure 2 Sagittal section of CT abdomen showing multiple growth of tumor.

\section{Vailbhav Pandey ${ }^{1}$, Anand Kumar Das², Indra Singh Choudhary ${ }^{2}$ and Om Prakash Sing ${ }^{3}$}

1 Department of Pediatric Surgery, Institute of Medical Sciences, Banaras Hindu University, India

2 Department of General Surgery, Institute of Medical Sciences, Banaras Hindu University, India

3 Department of Medicine, Institute of Medical Sciences, Banaras Hindu University, India

Corresponding authors:

Dr. Om Prakash Singh

$\equiv$ opbhu07@gmail.com

Department of Medicine, Institute of Medical Sciences, Banaras Hindu University, Varanasi - 221005, India.

Citation: Pandey V, Das AK, Choudhary IS, et al. A 2 Year Old Female Child with Huge Vaginal Rhabdomyosarcoma. Arch Cancer Res. 2016, 4:1. 